\title{
Digital images help toddlers with orall disease
}

Dental researchers are combining the ease of digital photography with the internet to develop a new, inexpensive way to screen for a common childhood oral disease that predominantly plagues America's inner city toddlers.

According to the University of Rochester Medical Center, USA, early childhood dental caries (ECC), or as it is commonly called, 'baby bottle tooth decay', where cavities are caused by prolonged exposure to sweetened juices often from sleeping with a bottle, tend to be overlooked by parents.

The pain can become so severe, and the teeth so decayed, that the only option for these toddlers - often under the age of four - is sedation and extraction. A specially outfitted digital camera is used to take photos of children's teeth by a child care centre health assistant. The photos are then sent electronically to paediatric dentists, who review the files in batches, identifying those toddlers with ECC.

Dentists believe that this new screening system is the first of its kind, and will pave the way for earlier identification of the cavities before they become a painful problem for young toddlers - and a costly one for states across the US footing the bill for Medicaid.

'We have identified a very simple, costeffective method to screen for this common childhood disease before it becomes a much larger problem,' said Dorota Kopycka-Kedzierawski, Assistant Professor of Dentistry and author of the study. 'By catching ECC at its earliest stage, we will effectively save the patient and parent toothache and heartache, decrease use of emergency room services, and increase the usage of dentists by this underserved population.'

In total, 162 children from one to five years of age in six Rochester inner-city child care centres were screened, with almost 40\% shown to have ECC. Once identified, parents received a letter alerting them to their child's oral disease, and were provided with a referral so the problem could be treated.

Three months later, all children were rescreened to determine how many had actually seen a dentist to correct the problem. About 25\% of children did receive dental services, though the sample size in this study is too small to make generalised conclusions. Now, researchers are focused on finding grants to help support follow-up efforts so that all toddlers identified with ECC will receive the much needed dental care.

\section{Hearing loss and high-speed dental tools}

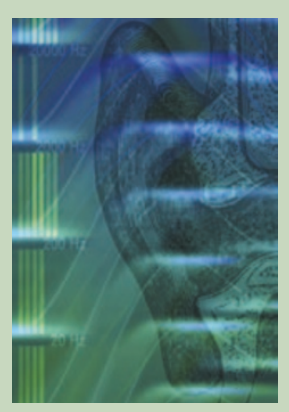

Researchers at Oregon Health and Science University, USA are looking into whether high-speed dental tools contribute to long-term hearing loss.

'Over the years, we have seen dentists in the OHSU Tinnitus Clinic who were convinced that long-term exposure to sound from highspeed hand pieces contributed to their high-frequency hearing loss and tinnitus,' said Professor Robert Folmer, one of the study leaders.

'These anecdotes, in combination with the research being divided about high-speed hand pieces playing a role in hearing loss, prompted our study. We hope the study is a good first step toward scientific evidence behind the anecdotes we've been hearing.'

Tinnitus, or ringing of the ears, can be constant or intermittent and can include buzzing, hissing or sizzling sounds. Many people experience momentary tinnitus, a high-pitched tone that lasts up to 30 seconds. Acute tinnitus, however, can last days or weeks and is most commonly caused by exposure to loud noise. The subsequent ringing indicates damage to the tiny hair-like structures within the inner ear and if exposure to loud noise continues, permanent hearing loss is likely.

Most of the current high-speed hand pieces, such as high-speed drills and scalers used by dental professionals, are between 90 to 100 decibels, says Professor Folmer. That's the equivalent of a gas lawnmower or other power tools, which are loud enough to cause hearing loss over time. He adds that while hand pieces have actually become 'quieter' over the years with the advent of modern technologies, very few dental professionals or students interviewed for the study so far wear earplugs to protect themselves from this noise.

The team will investigate noise-induced hearing loss by conducting hearing tests with a portable audiometer, examining ear canals with an otoscope, and asking participants to answer questions about occupational and recreational noise exposure. They also have measured the sound levels of different hand pieces while they're used on patients.

\section{Chewing gam with added bite}

A chewing gum that can destroy the bacteria that cause tooth decay could be in the shops soon according to the Society of Chemical Industry.

The gum is one of several products being developed by German chemical company BASF using the bacteria Lactobacillus, which is normally found in live yoghurt.

BASF has discovered a new strain of Lactobacillus called L. anti-caries, which binds to Streptococcus mutans, the bacteria responsible for tooth decay. S. mutans sticks to the surface of teeth, where it produces an aggressive acid that breaks down the enamel. The friendly bugs in the gum will make the S. mutans clump together, preventing them from becoming attached to the tooth surface. The company's tests reveal that the chewing gum can reduce the amount of bacteria in the mouth by 50 times.

Although the company has said a Lactobacillus product is due to hit the supermarkets in 2007, it will not confirm whether it is the chewing gum. A new range of toothpastes and mouthwashes using L. anti-caries is also in the pipeline. 